\title{
More Severe Erosive Phenotype Despite Lower Circulating Autoantibody Levels in Dipeptidyl Peptidase-4 Inhibitor (DPP4i)-Associated Bullous Pemphigoid: A Retrospective Cohort Study
}

\author{
Sascha Ständer ${ }^{1} \cdot$ Enno Schmidt $^{1,2} \cdot$ Detlef Zillikens ${ }^{1} \cdot$ Ralf J. Ludwig $^{1,2} \cdot$ Khalaf Kridin $^{2}$ (I)
}

Published online: 7 October 2020

(c) The Author(s) 2020

\begin{abstract}
Background The clinical and immunological profile of patients with dipeptidyl peptidase-4 inhibitor (DPP4i)-associated bullous pemphigoid (BP) is inconsistent in the current literature.

Objectives The aims were to investigate the clinical and immunological features of patients with DPP4i-associated BP and to examine whether there are intraclass differences between different DPP4i agents.

Methods A retrospective cohort study was conducted, including all consecutive patients diagnosed with BP throughout the years 2009-2019 in a tertiary referral center.

Results The study encompassed 273 patients with BP (mean age at diagnosis $79.1 \pm 9.9$ years), of whom 24 (8.8\%) were associated with DPP4i. Sitagliptin was the prescribed agent for 17 patients (70.8\%), and vildagliptin was prescribed in seven patients (29.2\%). Relative to other patients with BP, patients with DPP4i-associated BP had more prominent truncal involvement (95.8\% vs. 73.9\%; $P=0.017$ ), greater erosion/blister Bullous Pemphigoid Disease Area Index (BPDAI) subscore $(29.8 \pm 17.4$ vs. $20.6 \pm 14.4 ; P=0.018)$, and lower levels of anti-BP180 NC16A $(279.2 \pm 346.1 \mathrm{vs.} 572.2 \pm 1352.0 \mathrm{U} / \mathrm{ml}$; $P=0.009)$ and anti-BP230 (25.5 \pm 47.8 vs. $128.6 \pm 302.9 \mathrm{U} / \mathrm{ml} ; P=0.009)$ antibodies. Relative to patients with sitagliptinassociated BP, those with vildagliptin-associated $\mathrm{BP}$ had a lower seropositivity rate $(57.1 \%$ vs. $94.1 \%, P=0.031)$ and lower levels (96.7 \pm 139.0 vs. $354.5 \pm 376.5 ; P=0.023)$ of anti-BP180 NC16A antibodies, and tended to present with higher erosion/blister BPDAI subscore (36.3 \pm 9.6 vs. $25.8 \pm 19.7 ; P=0.095)$.
\end{abstract}

Conclusions DPP4i-associated BP is characterized by a more severe blistering and erosive presentation despite lower levels of typically pathogenic antibodies.

\section{Introduction}

Bullous pemphigoid (BP) is the most prevalent subepidermal autoimmune bullous disease in Western Europe and Northern America [1]. During the last 2 decades, a 1.9- to 4.3-fold rise in the incidence of BP was noted in France, Germany, and Israel, an epidemiological finding that has been attributed to multiple factors, including growing exposure to culprit medications [2]. Several lines of evidence

Ralf J. Ludwig and Khalaf Kridin contributed equally.

Khalaf Kridin

dr_kridin@hotmail.com

1 Department of Dermatology, University of Lübeck, Lübeck, Germany

2 Lübeck Institute of Experimental Dermatology, University of Lübeck, Ratzeburger Allee 160, 23562 Lübeck, Germany

\section{Key Points}

Patients with dipeptidyl peptidase-4 inhibitor-associated bullous pemphigoid (BP) demonstrate lower levels of anti-BP180 NC16A autoantibodies and a more severe erosive phenotype.

Patients with vildagliptin-associated BP had a lower seropositivity rate and lower levels of anti-BP180

NC16A antibodies compared with patients with sitagliptin-associated BP.

Given the inverse association between autoantibody levels and disease severity, it may be hypothesized that these patients target other epitopes or, alternatively, have a lower threshold to induce autoantibody-induced skin pathology in BP. 
suggested that dipeptidyl peptidase-4 inhibitors (DPP4i), or gliptins, a class of oral hypoglycemic drugs recently utilized to manage type 2 diabetes mellitus, may induce or aggravate BP. Data from a wide array of controlled epidemiological studies accumulated throughout the past few years to indicate that treatment with DPP4i is associated with an increased risk of developing BP [3-10].

Although exposure to DPP4i appears to be a risk factor of BP [11], it is yet to be delineated whether patients with DPP4i-associated BP possess a unique morphological, immunological, genetic, or histological profile distinguishing them from patients with classical BP [12-14]. While some studies observed distinct characteristics of this group of patients [6, 15-18], others refuted the presence of such characteristics [9, 19-21].

The primary aim of the current study was to evaluate the clinical and immunological characteristics of patients with DPP4i-associated BP relative to those of diabetic and nondiabetic patients with non-DPP4i-associated classical BP. The secondary aim was to assess the existence of intraclass differences between different DPP4i agents in terms of the clinical and immunological features of the associated BP.

\section{Methods}

\subsection{Study Population and Definition of Cases}

We performed a retrospective study comprising all patients diagnosed with BP between January 1st, 2009, and February 28th, 2020, at the Department of Dermatology, University of Lúbeck, Lúbeck, Germany. The current study was approved by the institutional ethical committee (20-110A).

The diagnosis of BP was based on the following criteria: (1) suggestive clinical picture; (2) linear deposits of $\operatorname{IgG}$ andor $\mathrm{C} 3$ along the dermal-epidermal junction by direct immunofluorescence (IF) microscopy of a perilesional skin biopsy; and (3) the detection of circulating autoantibodies binding to the epidermal side of $1 \mathrm{ml} \mathrm{NaCl}$-split normal human skin by indirect IF microscopy, andor the presence of circulating $\operatorname{IgG}$ autoantibodies against BP180 and/or BP230, as identified by BP180 NC16A/BP230 enzymelinked immunosorbent assay (ELISA) [22].

\subsection{Definition of Covariates}

The severity of disease was evaluated based on the Bullous Pemphigoid Disease Area Index (BPDAI) [23]. This score had been documented including its following subcomponents (erosion/blister activity, urticaria/erythema activity, damage, and pruritus). The non-inflammatory phenotype was defined in patients whose BPDAI urticaria/erythema score was zero [17].
The levels of anti-BP180 NC16A and anti-BP230 antibodies were measured using commercially available ELISA assays (Euroimmun, Lúbeck, Germany). The cutoff values proposed by the manufacturer (i.e., $20 \mathrm{U} / \mathrm{ml}$ ) were adopted for the definition of positivity.

\subsection{Statistical Analysis}

All continuous parameters were expressed as mean values (standard deviation [SD]). Percentages of different patient groups were compared using a Chi-square test. Normally distributed data were analyzed using the student $t$ test. Data found to be non-normally distributed were analyzed using the Mann-Whitney $U$ test for independent subgroups and the Wilcoxon test for dependent subgroups. SPSS software, version 25 (SPSS, Armonk, NY: IBM Corp) was utilized to conduct all statistical analyses.

\section{Results}

\subsection{Study Population}

The study cohort included 273 patients with BP, of whom 119 (43.6\%) were males, and 154 (56.4\%) females. The mean age (SD) at diagnosis was 79.1 (9.9) years, and the median age was 80.4 (range 49.6-98.2) years.

Overall, 75 patients (27.5\%) were diagnosed with type 2 diabetes mellitus at the onset of BP. Twenty-four patients (8.8\%) developed BP while being treated with DPP4i agents. Among those, the most frequently prescribed DPP4i was sitagliptin $(n=17 ; 70.8 \%)$, followed by vildagliptin $(n=7$; 29.2\%). No BP patients received another DPP4i agent.

\subsection{Characterization of Patients with DPP4i-Associated BP}

The primary endpoint of the current study was to address the differences between patients receiving DPP4 $\mathrm{i}$ at the diagnosis of BP $(n=24)$ relative to BP patients without DPP4i exposure $(n=249)$. No significant differences were observed between the subgroups with respect to the age of presentation and sex distribution (Table 1).

Regarding the anatomical distribution of bullous lesions, patients with DPP4i-associated BP had greater truncal involvement (95.8\% vs. $73.9 \% ; P=0.017)$. No significant differences were observed between the two subgroups regarding the involvement of other body sites or the prevalence of the non-inflammatory phenotype of BP. Patients were additionally compared in terms of their BPDAI scores. Patients with DPP4i-associated BP were found to have a higher blister/erosion BPDAI mean (SD) score (29.8 [17.4] vs. 20.6 [14.4], respectively; $P=0.018$; Fig. 1a). The 
Table 1 Demographic, clinical, and immunological characteristics of patients with DPP4i-associated BP compared with BP patients not taking DPP4i

DPP4i-associated BP $(n=24) \quad$ Non-DPP4i-associated BP $\quad P$ value $(n=249)$

\begin{tabular}{|c|c|c|c|}
\hline \multicolumn{4}{|l|}{ Age at diagnosis; years } \\
\hline Mean (SD) & $77.48(6.4)$ & $79.20(10.2)$ & \multirow[t]{2}{*}{0.247} \\
\hline Median (range) & $77.74(61.5-89.7)$ & $80.65(49.6-98.2)$ & \\
\hline \multicolumn{4}{|l|}{ Sex, $n(\%)$} \\
\hline Male & $11(45.8)$ & $108(43.4)$ & \multirow[t]{2}{*}{0.813} \\
\hline Female & $13(54.2)$ & $141(56.6)$ & \\
\hline \multicolumn{4}{|l|}{ Distribution of bullous lesions, $n(\%)$} \\
\hline Limbs & $18(75.0)$ & $213(85.5)$ & 0.175 \\
\hline Trunk & $23(95.8)$ & $184(73.9)$ & 0.017 \\
\hline Hands/feet & $11(45.8)$ & $100(40.2)$ & 0.595 \\
\hline Head and neck & $7(29.2)$ & $68(27.3)$ & 0.842 \\
\hline Mucosal involvement & $3(12.5)$ & $28(11.2)$ & 0.848 \\
\hline Non-inflammatory phenotype, $n(\%)^{*}$ & $1(6.3)$ & $16(13.8)$ & 0.403 \\
\hline \multicolumn{4}{|l|}{ Mean BPDAI severity score (SD)* } \\
\hline Erosion/blister activity & $29.8(17.4)$ & $20.6(14.4)$ & $\mathbf{0 . 0 1 8}$ \\
\hline Urticaria/erythema activity & $12.5(6.8)$ & $12.5(16.0)$ & 0.999 \\
\hline Damage score & $2.2(3.6)$ & $2.1(3.1)$ & 0.896 \\
\hline Pruritus score & $20.3(10.1)$ & $19.1(8.9)$ & 0.627 \\
\hline \multicolumn{4}{|l|}{ Anti-BP180 NC16A ELISA** } \\
\hline Seropositivity, $n(\%)$ & $20(83.3)$ & $201(82.7)$ & 0.940 \\
\hline ELISA value, mean (SD); U/ml & $279.2(346.1)$ & $572.2(1352.0)$ & 0.009 \\
\hline \multicolumn{4}{|l|}{ Anti-BP230 ELISA*** } \\
\hline Seropositivity, $n(\%)$ & $3(30.0)$ & $38(52.1)$ & 0.193 \\
\hline ELISA value, mean (SD); U/ml & $25.5(47.8)$ & $128.6(302.9)$ & 0.009 \\
\hline
\end{tabular}

Significant values are shown in bold

Anti-BP180 NC16A and anti-BP230 antibodies levels were measured via ELISA; cutoff: $20.0 \mathrm{U} / \mathrm{ml}$

$B P$ bullous pemphigoid, BPDAI Bullous Pemphigoid Disease Area Index, DPP4i dipeptidyl peptidase-4 inhibitor(s), ELISA enzyme-linked immunosorbent assay, $n$ number, $S D$ standard deviation

*Was calculated for 16 patients with DPP4i-associated BP and 116 patients with non-DPP4i-associated BP

**Was performed in all patients with DPP4i-associated BP and in 243 patients with non-DPP4i-associated BP

***Was performed in 10 patients with DPP4i-associated BP and in 73 patients with non-DPP4i-associated BP

remaining components of the BPDAI score [namely, urticaria/erythema (Fig. 1b), damage, and pruritus] were comparable between the two subgroups (Table 1).

Overall, 267 (97.8\%) of patients were tested for the presence of circulating anti-BP180 NC16A antibodies. While the detection rate of anti-BP180 NC16A antibodies was comparable between the two subgroups, patients with DPP4iassociated BP had significantly lower mean (SD) levels of these antibodies $(279.2$ [346.1] vs. $572.2[1352.0] \mathrm{U} / \mathrm{ml}$, respectively; $P=0.009$; Fig. 2 ). Additionally, 83 patients $(30.4 \%)$ were tested for anti-BP230 antibodies. The mean (SD) anti-BP230 levels were also lower among patients with DPP4i-associated BP (25.2 [47.8] vs. 128.6 [302.9] $\mathrm{U} / \mathrm{ml}$, respectively; $P=0.009$ ), while the seropositivity of these antibodies tended to be lower in this subgroup (30\% vs. $52.1 \%$, respectively; $P=0.193$; Table 1 ).

To exclude any confounding factor through the additional diagnosis of diabetes mellitus, we next studied the difference between diabetic patients with DPP4i-associated $\mathrm{BP}(n=24)$ relative to diabetic patients with non-DPP4iassociated BP $(n=51)$. While no morphological discrepancies appeared between the two subgroups, the former group again had lower levels of circulating anti-BP180 NC16A antibodies (279.2 [346.1] vs. 696.2 [1340.1] U/ $\mathrm{ml} ; P=0.045)$, lower positivity rate $(30.0 \%$ vs. $81.3 \%$; $P=0.010)$ as well as lower levels (25.5 [47.8] vs. 211.4 [330.3] U/ml; $P=0.042$ ) of anti-BP230 antibodies. The mean blister/erosion BPDAI score tended to be higher 
Fig. 1 Values of erosion/blister (a) and urticaria/erythema (b) BPDAI scores among patients with DPP4i-associated BP vs. non-DPP4i-associated BP. $B P$ bullous pemphigoid, $B P D A I$ Bullous Pemphigoid Disease Area Index, DPP4i dipeptidyl peptidase-4 inhibitor
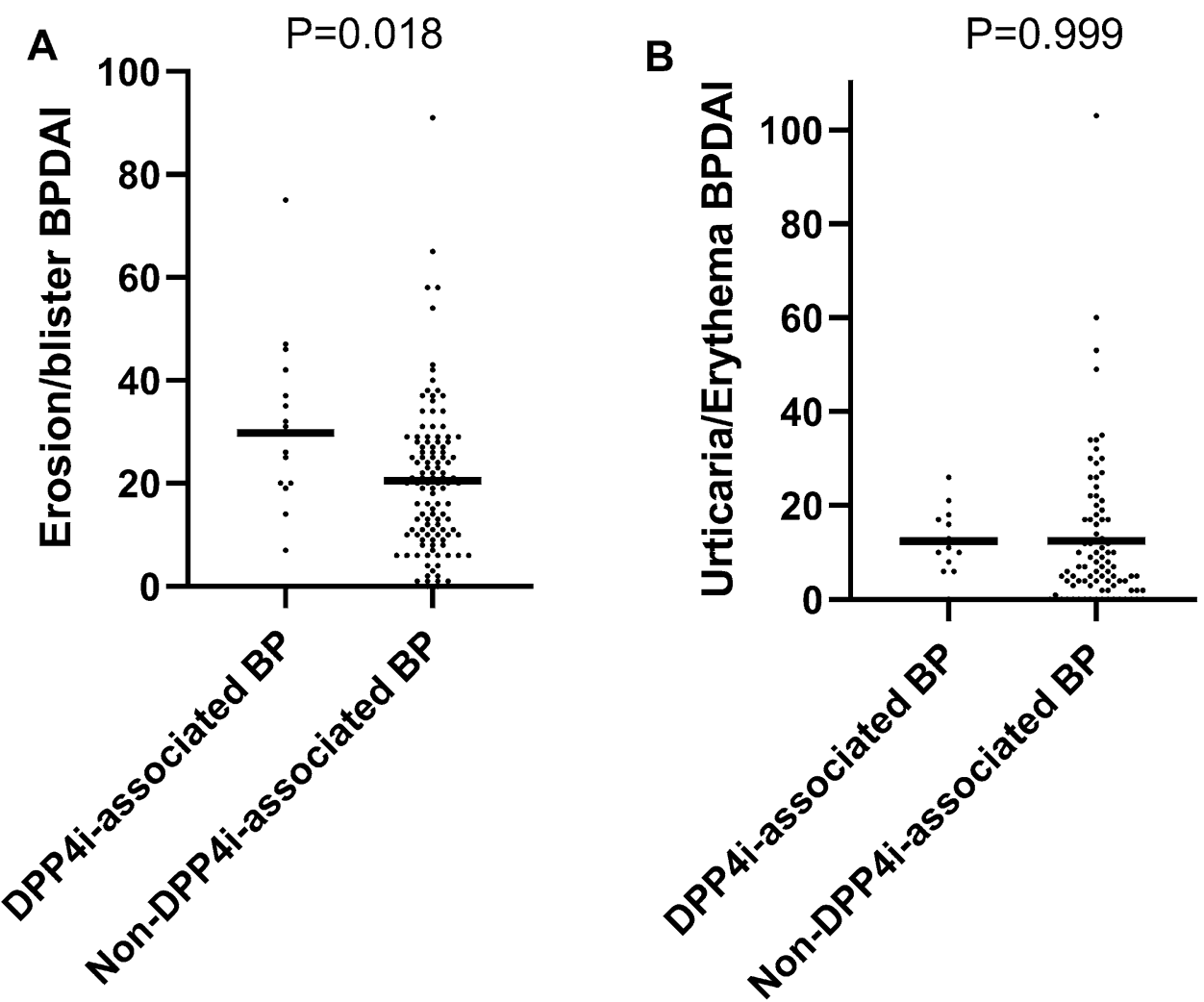

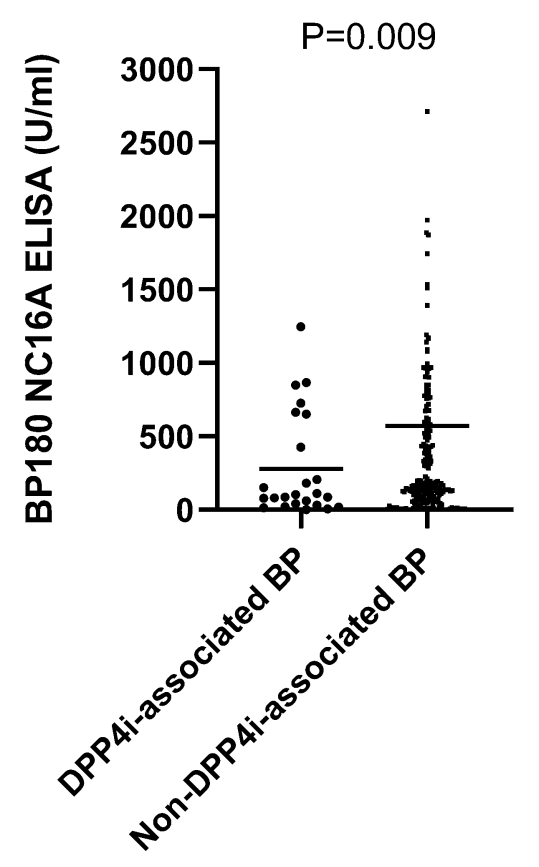

Fig. 2 Levels of anti-BP180 NC16A antibodies among patients with DPP4i-associated BP vs. non-DPP4i-associated BP. Rules represent mean values. $B P$ bullous pemphigoid, $D P P 4 i$ dipeptidyl peptidase- 4 inhibitor among patients with DPP4i-associated BP, although it lacked statistical significance (Table 2).

To refute differential effect exerted by other anti-diabetic medications, the two subgroups were compared with regard to exposure to non-DPP4i anti-diabetic medications. Out of patients with DPP4i-associated BP, 17 (70.8\%) were managed by additional anti-diabetic medications, whereas 38 diabetic patients with non-DPP4i-associated BP (74.5\%) had an exposure to these medications $(P=0.737)$. The two subgroups were comparable with regard to exposure to metformin ( $45.8 \%$ vs. $33.3 \%$, respectively; $P=0.299$ ) and insulin ( $45.8 \%$ vs. $47.1 \%$, respectively; $P=0.917)$.

\subsection{Identification of Intraclass Variations}

Our secondary endpoint was to evaluate intraclass differences in the clinical and immunological features of BP associated with different DPP4i agents. A comparison was undertaken between patients with BP under the two encountered agents: sitagliptin $(n=17)$ and vildagliptin $(n=7$; Table 3).

Patients with sitagliptin-associated BP had a higher seropositivity rate $(94.1 \%$ vs. $57.1 \%, P=0.031)$ and higher mean (SD) levels (354.5 [376.5] vs. 96.7 [139.0] U/ml; $P=0.023)$ of anti-BP180 NC16A antibodies. A tendency towards a greater mean (SD) erosion/blister BPDAI score was observed in patients with vildagliptin-associated BP 
(36.3 [9.6] vs. 25.8 [19.7]), albeit it did not reach the level of statistical significance $(P=0.095$; Table 3$)$.

\section{Discussion}

Our current study revealed that patients with DPP4i-associated BP are characterized by more predominant truncal involvement, a more severe blistering phenotype (as reflected by erosion/blister BPDAI scores), and significantly lower serum levels of anti-BP180 NC16A and anti-BP230 autoantibodies. Compared to patients with vildagliptin-associated BP, those with sitagliptin-associated BP had a higher positivity rate and higher levels of anti-BP180 NC16A antibodies.
Despite the accumulating data on the association of BP with DPP4i, there remains a debate around the clinical and immunological profile of patients with DPP4i-associated BP. This inconclusiveness stems mainly from the variable findings observed in different studies. Notable differences were mainly observed between studies from Japan attributing distinct features (like non-inflammatory phenotype and targeting atypical epitopes along BP180) to Japanese patients on one side and studies reporting typical features in European patients on the other. Table 4 summarizes the main findings of the studies carried out so far to investigate the different features of patients with DPP4i-associated BP.

Unlike other studies reporting a male predominance among patients with DPP4i-associated BP [3, 6, 8, 24], the sex distribution in our cohort was similar between the two

Table 2 Demographic, clinical, and immunological characteristics of patients with DPP4i-associated BP compared with diabetic patients not taking DPP4i

\begin{tabular}{|c|c|c|c|}
\hline & DPP4i-associated BP $(n=24)$ & $\begin{array}{l}\text { Non-DPP4i-associated diabetic BP } \\
(n=51)\end{array}$ & $P$ value \\
\hline \multicolumn{4}{|l|}{ Age at diagnosis; years } \\
\hline Mean (SD) & $77.5(6.4)$ & $79.7(8.8)$ & 0.225 \\
\hline \multicolumn{4}{|l|}{ Sex, $n(\%)$} \\
\hline Male & $11(45.8)$ & $29(56.9)$ & 0.372 \\
\hline Female & $13(54.2)$ & $22(43.1)$ & \\
\hline \multicolumn{4}{|l|}{ Distribution of bullous lesions; $n(\%)$} \\
\hline Limbs & $18(75.0)$ & $46(90.2)$ & 0.175 \\
\hline Trunk & $23(95.8)$ & $38(74.5)$ & 0.085 \\
\hline Hands/feet & $11(45.8)$ & $21(41.2)$ & 0.709 \\
\hline Head and neck & $7(29.2)$ & $19(37.3)$ & 0.495 \\
\hline Mucosal involvement & $3(12.5)$ & $4(7.8)$ & 0.516 \\
\hline Non-inflammatory phenotype, $n(\%)^{*}$ & $1(6.3)$ & $6(21.4)$ & 0.192 \\
\hline \multicolumn{4}{|l|}{ Mean BPDAI severity score (SD)* } \\
\hline Erosions/blister activity & $29.8(17.4)$ & $23.4(14.9)$ & 0.128 \\
\hline Urticaria/erythema activity & $12.5(6.8)$ & $8.7(11.7)$ & 0.145 \\
\hline Damage score & $2.2(3.6)$ & $2.4(3.6)$ & 0.824 \\
\hline Pruritus score & $20.3(10.1)$ & $18.8(8.4)$ & 0.531 \\
\hline \multicolumn{4}{|l|}{ Anti-BP180 NC16A ELISA** } \\
\hline Seropositivity, $n(\%)$ & $20(83.3)$ & $43(87.8)$ & 0.602 \\
\hline ELISA value, mean (SD); U/ml & $279.2(346.1)$ & $696.2(1340.1)$ & 0.045 \\
\hline \multicolumn{4}{|l|}{ Anti-BP230 ELISA*** } \\
\hline Seropositivity, $n(\%)$ & $3(30.0)$ & $13(81.3)$ & 0.010 \\
\hline ELISA value, mean (SD); U/ml & $25.5(47.8)$ & $211.4(330.3)$ & 0.042 \\
\hline
\end{tabular}

Significant values are shown in bold

Anti-BP180 NC16A and anti-BP230 antibodies levels were measured via ELISA; cutoff: $20.0 \mathrm{U} / \mathrm{ml}$

$B P$ bullous pemphigoid, BPDAI Bullous Pemphigoid Disease Area Index, DPP4i dipeptidyl peptidase-4 inhibitor(s), ELISA enzyme-linked immunosorbent assay, $n$ number, $S D$ standard deviation

*Was calculated for 16 patients with DPP4i-associated BP and for 28 diabetic patients with non-DPP4i-associated BP

**Was performed in all patients with DPP4i-associated BP and in 49 diabetic patients with non-DPP4i-associated BP

***Was performed in 10 patients with DPP4i-associated BP and in 16 diabetic patients with non-DPP4i-associated BP 
Table 3 Demographic, clinical, and immunological characteristics of sitagliptin-associated BP compared with vildagliptin-associated BP patients

\begin{tabular}{lll}
\hline Characteristic & Sitagliptin-associated BP $(n=17)$ & Vildagliptin-associated BP $(n=7)$ \\
\hline Age at diagnosis; years & & $P$ value \\
Mean (SD) & $78.1(6.1)$ & $75.9(7.0)$ \\
Sex, $n$ (\%) & $9(52.9)$ & $2(28.6)$ \\
Male & $8(47.1)$ & $5(71.4)$ \\
Female & & \\
Mean BPDAI severity score (SD)* & $25.8(19.7)$ & $36.3(9.6)$ \\
Erosions/blister activity & $11.1(6.1)$ & $15.5(7.2)$ \\
Urticaria/erythema activity & $2.3(3.6)$ & $2.0(3.5)$ \\
Damage score & & $4(57.1)$ \\
Anti-BP180 NC16A ELISA & $16(94.1)$ & $96.7(139.0)$ \\
Seropositivity, $n$ (\%) & $354.5(376.5)$ & 0.288 \\
ELISA value, mean (SD); U/ml & $2(33.3)$ & $1(25.0)$ \\
Anti-BP230 ELISA** & $37.0(58.7)$ & $8.3(7.6)$ \\
Seropositivity, $n$ (\%) & & 0.186 \\
ELISA value, mean (SD); U/ml & & $\mathbf{0 . 0 3 1}$ \\
\hline
\end{tabular}

Significant values are shown in bold

Anti-BP180 NC16A and anti-BP230 antibodies levels were measured via ELISA; cutoff: $20 \mathrm{U} / \mathrm{ml}$

$B P$ bullous pemphigoid, BPDAI Bullous Pemphigoid Disease Area Index, DPP4i dipeptidyl peptidase-4 inhibitor(s), ELISA enzyme-linked immunosorbent assay, $n$ number, $S D$ standard deviation

*Was calculated for 10 patients with sitagliptin-associated BP and for 6 patients with vildagliptin-associated BP

**Was performed in 6 patients with sitagliptin-associated BP and in 4 patients with vildagliptin-associated BP

subgroups, in line with two studies from Finland [4] and France [9].

The non-inflammatory phenotype was a prominent morphological feature among Japanese patients with DPP4iassociated BP, where its prevalence ranged between 50 and $70 \%$ [16-18]. This finding was not reproduced in studies tracking Caucasian patients, where the non-inflammatory phenotype was less frequent (40\% in Finland [19], 0\% in Italy [21], and $6.3 \%$ in the current study). A clinicopathological correlation was reported in Japanese patients demonstrating that the non-inflammatory phenotype was paralleled by an only scant eosinophilic dermal infiltrate in perilesional skin $[15,16,18]$. A study from Israel lent weight to this finding by detecting lower circulating eosinophil counts among these patients [6]. On the contrary, two European studies refuted the existence of a distinct histological pattern by revealing a comparable dermal eosinophil count in patients with DPP4i-associated BP relative to their non-DPP4I-associated BP counterparts (Table 4) [19, 20]. To shed further light on ethnic variations, a recent American study utilized two large commercial insurance claims databases to follow patients placed on DPP4i and second-generation sulfonylureas. This study revealed an increased risk of BP in the former groups, with White individuals possessing a higher risk than their non-white counterparts [10]. Unlike a previous study attributing a higher frequency of mucosal lesions in patients with DPP4i-associated BP [6, 25], mucosal involvement among our DPP4i-associated patients was as common as among the remaining patients.

The current study suggests that patients with DPP4i-associated BP had a more severe bullous component, as indicated by a higher erosion/blister BPDAI score. This finding is in accordance with an Israeli study evaluating 58 patients with DPP4i-associated BP, who presented with significantly more extensive disease [26]. Additionally, Patsatsi et al. [20] revealed that patients with DPP4i-associated BP had higher total BPDAI scores, with a trend towards significance (41.0 vs. $34.1 ; P=0.063$ ). Similarly, Horikawa et al. [17] and Chijiwa et al. [15] demonstrated that the erosion/blister component of the BPDAI score tended to be higher among patients with DPP4i-associated BP, albeit lacking statistical significance. Given the relatively small sample size of these studies, one may assume that they were underpowered to provide differences of statistical significance; still, they reflected a trend towards a more severe phenotype.

Immunologically, we found that patients with DPP4iassociated BP had significantly lower levels of anti-BP180 NC16A antibodies. This finding held significance also when comparing this subgroup to diabetic patients with non-DPP4i-associated BP, thus refuting major confounding conferred by diabetes mellitus itself. While this finding 


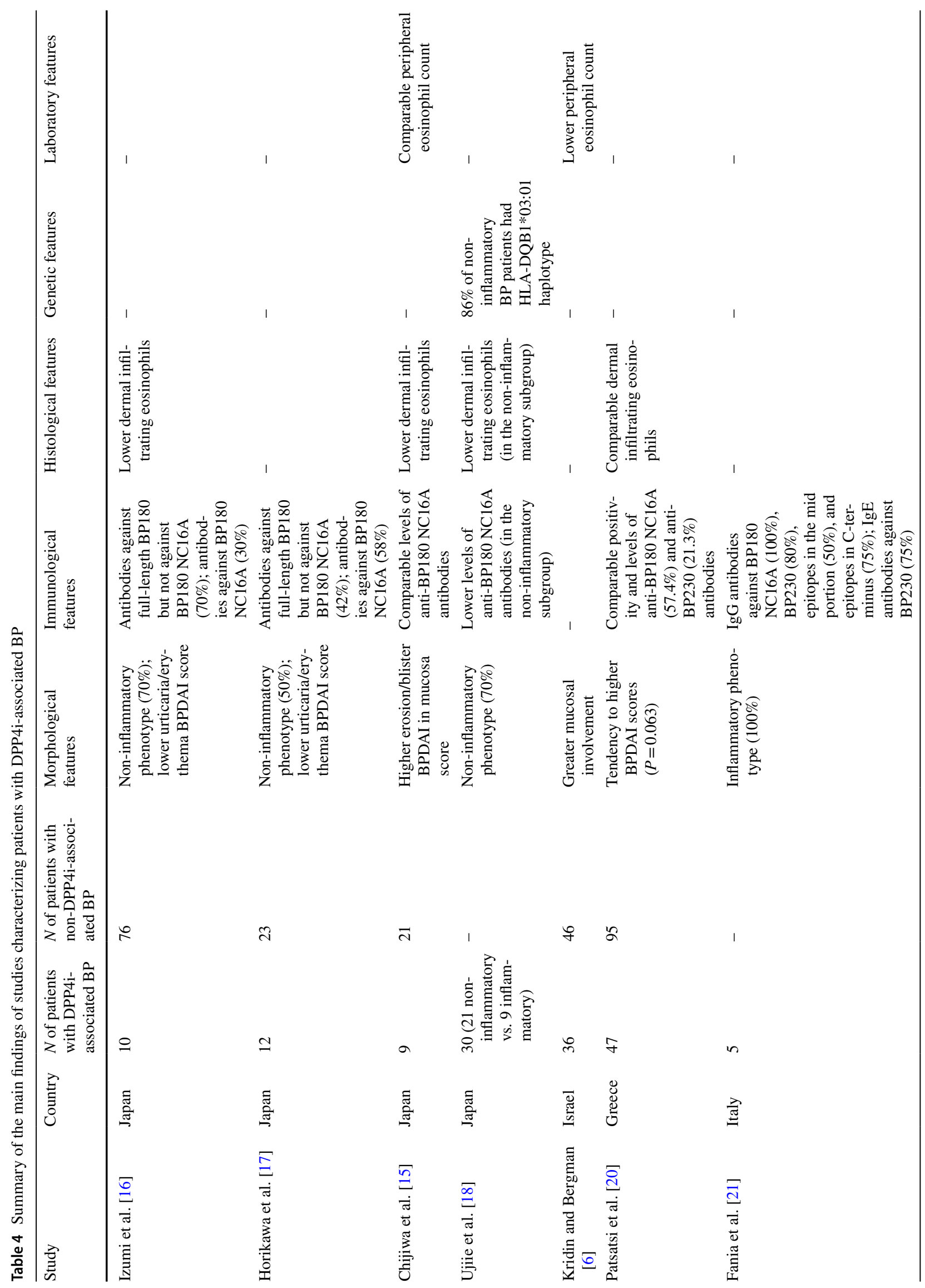




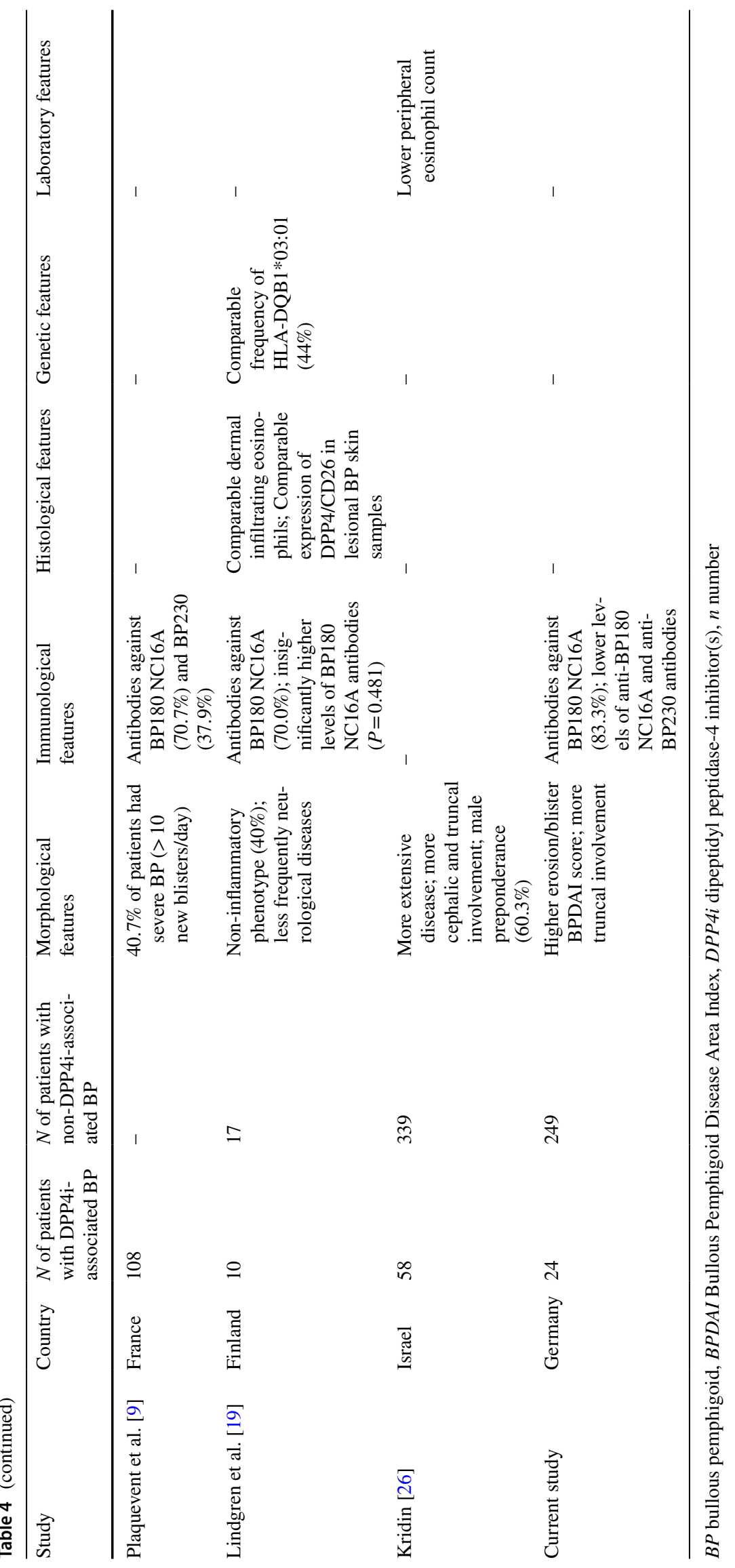


aligns with a previous study by Ujiie et al. [18], it is not consistent with the studies of Patsatsi et al. [20], Chijiwa et al. [15], and Lindgren et al. [19] reporting similar levels of these antibodies in DPP4i-associated BP and non-DPP4iassociated BP. The positivity rate of anti-BP180 NC16A antibodies in the current study was estimated at $83.3 \%$ among patients with DPP4i-associated BP. While earlier studies, originating mainly from Japan, disclosed low seropositivity of the aforementioned antibodies ranging between 30 and 58\% [16, 17, 20], later European studies have shown higher detection rates, ranging from 70 to $100 \%$ [9, 19, 21], a range which our figure falls within (Table 3 ). In a recent Japanese cross-sectional study examining sera from type 2 diabetes mellitus patients with and without DPP4i exposure, the former group had higher levels of anti-full-length BP180, but not anti-BP180 NC16A and anti-BP230 IgG [27]. The latter finding may lend credibility to our observation regarding the lower levels of anti-BP180 NC16A. In a recent study of 18 Japanese DPP4i-associtaed BP patients targeting the non-NC16A domains of BP180, Mai et al. [28] revealed that the all sera reacted more intensively with the $97-\mathrm{kDa}$ processed extracellular domain of BP180 (LABD97) autoantigen than with full-length BP180. Thus, anti-LABD97 IgG1 represented the major autoantibodies in these DPP4i-associated BP patients.

The detection rate of anti-BP230 antibodies in this study (30\%) compares with the figures reported by Patsatsi et al. [20] (21.3\%) and Plaquevent et al. [9] (37.9\%). The relative serum levels of this autoantibody had been assessed only by Patsatsi et al. [20] and were found to be similar between patients with DPP4i-associated BP relative to those with typical BP. In contrast, we found decreased levels in the current study.

Taken together, in our cohort of $273 \mathrm{BP}$ patients, those with DPP4i-associated BP presented with a more severe erosive disease despite generating lower levels of autoantibodies (both anti-NC16A BP180 and anti-BP230 IgG). This intriguing finding may lead us to postulate that patients with this disease target other epitopes along the BP180 autoantigen, which are not detected by the commercially available ELISA. Small-scale Japanese studies assumed that these patients synthesize autoantibodies against epitopes along the midportion of the extracellular domain of BP180, but not against the NC16A domain $[16,17]$. We were not able to examine this hypothesis since our retrospective analysis merely evaluated the presence of $\operatorname{IgG}$ autoantibodies against the immunodominant NC16A domain of BP180. Additional immunoblotting was performed in a few of our patients, but did not enable us to draw meaningful conclusions. Alternatively, autoantibodies from DPP4i-associated BP may have other, more pro-inflammatory properties, i.e., a G0 IgG glycosylation pattern that was shown to promote skin inflammation [29].
It is still unknown whether different DPP4i agents trigger BP with different clinical and immunological features. Interestingly, we observed that patients with vildagliptinassociated BP had a lower seropositivity rate of antibodies targeting the immunodominant domain of BP180 and exhibited lower levels of these antibodies. However, despite their decreased production of detectable autoantibodies, these patients tended to present with a more widespread blistering/erosive phenotype as compared with their sitagliptinassociated counterparts. This substantiates the assumption that patients with DPP4i-associated BP, particularly those with vildagliptin-associated BP, either target other yet unknown epitopes, or generate highly pathogenic autoantibodies. Although the highest risk of DPP4i-associated BP was ascribed to vildagliptin [11], patients with sitagliptinassociated BP were more frequently encountered than their vildagliptin-associated counterparts in our study. The latter finding probably reflects the more common usage of sitagliptin in Germany.

The main limitation of the current study is its retrospective design, which enabled the collection of data of immunoassays utilized for the purpose of fulfilling diagnostic criteria, but not for research purposes. However, the routine immunological work-up at our department is very comprehensive, thus facilitating the characterization of the immunological profile of patients with DPP4i-associated BP. Selection bias may have arisen because the study was performed in tertiary-care referral center settings, rendering it susceptible to overlooking mild BP cases managed in outpatient community settings, Although the general study population was relatively high, some subgroup analyses were based on low numbers of patients.

The current study sheds light on a topic with inconclusive findings reported in the current literature so far. Moreover, it provides novel insights regarding intraclass variations between different DPP4i agents. The routinely collected clinical and immunoserological variables enabled relatively broad profiling of the patients.

\section{Conclusion}

In conclusion, we show that patients developing BP under DPP4i treatment are characterized by a more severe erosive phenotype and by more prominent truncal involvement. Immunologically, these patients have lower levels of anti-BP180 NC16A and anti-BP230 autoantibodies. Patients with vildagliptin-associated BP have an even lower prevalence of these specific autoantibodies, decreased levels of anti-NC16A BP180 IgG, and tended to show a more severe erosive phenotype. Given the lack of correlation between clinical disease severity and the routinely examined autoantibody levels, our findings indicate that patients 
with DPP4i-associated BP produce autoantibodies against other epitopes than those with non-DPP4i-associated BP or, alternatively, that DPP4i lead to the production of more proinflammatory autoantibodies.

\section{Declarations}

Funding Open Access funding provided by Projekt DEAL. Clinical Research Unit Pemphigoid Diseases (KFO 303) and Cluster of Excellence Precision Medicine in Chronic Inflammation (EXC 2167) are both funded by Deutsche Forschungsgemeinschaft.

Conflict of interest Sascha Ständer, Enno Schmidt, Detlef Zillikens, Ralf J. Ludwig, and Khalaf Kridin have no conflicts of interest to declare.

Ethical approval The current study was approved by the appropriate institutional research ethics committee of the University of Lübeck (20-110A). The study was performed in accordance with the ethical standards as laid down in the 1964 Declaration of Helsinki and its later amendments or comparable ethical standards.

Consent to participate Not applicable.

Consent for publication Not applicable.

Availability of data and material Raw data are available on request from the authors.

\section{Code availability Not applicable.}

Author contributions KK and SS had full access to all of the data in the study and take responsibility for the integrity of the data and the accuracy of the data analysis. Study concept and design: KK and RJL. Acquisition, analysis, and interpretation of data: KK and RJL. Drafting of the manuscript: KK. Critical revision of the manuscript for important intellectual content: ES and DZ. Statistical analysis: KK. Study supervision: SS. All authors have read and approved the manuscript.

Open Access This article is licensed under a Creative Commons Attribution-NonCommercial 4.0 International License, which permits any non-commercial use, sharing, adaptation, distribution and reproduction in any medium or format, as long as you give appropriate credit to the original author(s) and the source, provide a link to the Creative Commons licence, and indicate if changes were made. The images or other third party material in this article are included in the article's Creative Commons licence, unless indicated otherwise in a credit line to the material. If material is not included in the article's Creative Commons licence and your intended use is not permitted by statutory regulation or exceeds the permitted use, you will need to obtain permission directly from the copyright holder. To view a copy of this licence, visit http://creativecommons.org/licenses/by-nc/4.0/.

\section{References}

1. Kridin K. Subepidermal autoimmune bullous diseases: overview, epidemiology, and associations. Immunol Res. 2018;66(1):6-17. https://doi.org/10.1007/s12026-017-8975-2.

2. Kridin K, Ludwig RJ. The growing incidence of bullous pemphigoid: overview and potential explanations. Front Med. 2018;5:220. https://doi.org/10.3389/fmed.2018.00220.
3. Benzaquen M, Borradori L, Berbis P, Cazzaniga S, Valero R, Richard M-A, et al. Dipeptidyl peptidase IV inhibitors, a risk factor for bullous pemphigoid: retrospective multicenter case-control study from France and Switzerland. J Am Acad Dermatol. 2018;78(6):1090-6. https://doi.org/10.1016/j.jaad.2017.12.038.

4. Varpuluoma O, Försti A-K, Jokelainen J, Turpeinen M, Timonen M, Huilaja L, et al. Vildagliptin significantly increases the risk of bullous pemphigoid: a Finnish nationwide registry study. J Invest Dermatol. 2018;138(7):1659-61. https://doi.org/10.1016/j. jid.2018.01.027.

5. Schaffer C, Buclin T, Jornayvaz FR, Cazzaniga S, Borradori L, Gilliet M, et al. Use of dipeptidyl-peptidase IV inhibitors and bullous pemphigoid. Dermatology. 2017;233(5):401-3. https:// doi.org/10.1159/000480498.

6. Kridin K, Bergman R. Association of bullous pemphigoid with dipeptidyl-peptidase 4 inhibitors in patients with diabetes. JAMA Dermatol. 2018;154(10):1152-8. https://doi.org/10.1001/jamad ermatol.2018.2352.

7. Douros A, Rouette J, Yin H, Yu OHY, Filion KB, Azoulay L. Dipeptidyl peptidase 4 inhibitors and the risk of bullous pemphigoid among patients with type 2 diabetes. Diabetes Care. 2019;42(8):1496-503. https://doi.org/10.2337/dc19-0409.

8. Lee SG, Lee HJ, Yoon MS, Kim DH. Association of dipeptidyl peptidase 4 inhibitor use with risk of bullous pemphigoid in patients with diabetes. JAMA Dermatol. 2019;155(2):172-7. https ://doi.org/10.1001/jamadermatol.2018.4556.

9. Plaquevent M, Tétart F, Fardet L, Ingen-Housz-Oro S, ValeyrieAllanore L, Bernard P, et al. Higher frequency of dipeptidyl peptidase-4 inhibitor intake in bullous pemphigoid patients than in the french general population. J Invest Dermatol. 2019;139(4):83541. https://doi.org/10.1016/j.jid.2018.10.045.

10. Lee H, Chung HJ, Pawar A, Patorno E, Kim DH. Evaluation of risk of bullous pemphigoid with initiation of dipeptidyl peptidase-4 inhibitor vs second-generation sulfonylurea. JAMA Dermatol. 2019;155(2):172-7. https://doi.org/10.1001/jamadermat ol.2018.4556;

11. Kridin K, Cohen AD. Dipeptidyl-peptidase IV inhibitor-associated bullous pemphigoid: a systematic review and meta-analysis. J Am Acad Dermatol. 2018. https://doi.org/10.1016/j.jaad.2018.09.048.

12. Nishie W, Tasanen K. Gliptin-associated bullous pemphigoid: a valuable model of the mechanism of breakdown of immune tolerance against BP180. J Invest Dermatol. 2019;139(4):755-6. https ://doi.org/10.1016/j.jid.2018.11.025.

13. Nishie W. Dipeptidyl peptidase IV inhibitor-associated bullous pemphigoid: a recently recognized autoimmune blistering disease with unique clinical, immunological and genetic characteristics. Immunol Med. 2019;42(1):22-8. https://doi.org/10.1080/25785 826.2019 .1619233$.

14. Tasanen K, Varpuluoma O, Nishie W. Dipeptidyl peptidase-4 inhibitor-associated bullous pemphigoid. Front Immunol. 2019;10:1238. https://doi.org/10.3389/fimmu.2019.01238.

15. Chijiwa C, Takeoka S, Kamata M, Tateishi M, Fukaya S, Hayashi K, et al. Decrease in eosinophils infiltrating into the skin of patients with dipeptidyl peptidase-4 inhibitor-related bullous pemphigoid. J Dermatol. 2018;45(5):596-9. https://doi. org/10.1111/1346-8138.14245.

16. Izumi K, Nishie W, Mai Y, Wada M, Natsuga K, Ujiie H, et al. Autoantibody profile differentiates between inflammatory and noninflammatory bullous pemphigoid. J Invest Dermatol. 2016;136(11):2201-10. https://doi.org/10.1016/j.jid.2016.06.622.

17. Horikawa H, Kurihara Y, Funakoshi T, Umegaki-Arao N, Takahashi $\mathrm{H}$, Kubo A, et al. Unique clinical and serological features of bullous pemphigoid associated with dipeptidyl peptidase- 4 inhibitors. Br J Dermatol. 2018;178(6):1462-3. https://doi.org/10.1111/ bjd.16479. 
18. Ujiie H, Muramatsu K, Mushiroda T, Ozeki T, Miyoshi H, Iwata $\mathrm{H}$, et al. HLA-DQB1*03:01 as a biomarker for genetic susceptibility to bullous pemphigoid induced by DPP-4 inhibitors. J Invest Dermatol. 2018;138(5):1201-4. https://doi.org/10.1016/j. jid.2017.11.023.

19. Lindgren O, Varpuluoma O, Tuusa J, Ilonen J, Huilaja L, Kokkonen N, et al. Gliptin-associated bullous pemphigoid and the expression of dipeptidyl peptidase-4/CD26 in bullous pemphigoid. Acta Derm Venereol. 2019;99(6):602-9. https://doi. org/10.2340/00015555-3166.

20. Patsatsi A, Kyriakou A, Meltzanidou P, Trigoni A, Lamprou F, Kokolios M, et al. Bullous pemphigoid in patients with DPP-4 inhibitors at the onset of disease: does this differ from common bullous pemphigoid? Eur J Dermatol. 2018;28(5):711-3. https:// doi.org/10.1684/ejd.2018.3371.

21. Fania L, Salemme A, Provini A, Pagnanelli G, Collina MC, Abeni D, et al. Detection and characterization of IgG, IgE, and IgA autoantibodies in patients with bullous pemphigoid associated with dipeptidyl peptidase-4 inhibitors. J Am Acad Dermatol. 2018;78(3):592-5.

22. Schmidt E, Goebeler M, Hertl M, Sardy M, Sitaru C, Eming R, et al. S2k-Leitlinie zur Diagnostik des Pemphigus vulgaris/foliaceus und des bullösen Pemphigoids. JDDG J German Soc Dermatol. 2020;18(5):516-27. https://doi.org/10.1111/ddg.14097_g.

23. Murrell DF, Daniel BS, Joly P, Borradori L, Amagai M, Hashimoto T, et al. Definitions and outcome measures for bullous pemphigoid: recommendations by an international panel of experts. $\mathrm{J}$ Am Acad Dermatol. 2012;66(3):479-85. https://doi.org/10.1016/j. jaad.2011.06.032.
24. Béné J, Moulis G, Bennani I, Auffret M, Coupe P, Babai S, et al. Bullous pemphigoid and dipeptidyl peptidase IV inhibitors: a case-noncase study in the French Pharmacovigilance Database. Br J Dermatol. 2016;175:296-301.

25. Kridin K, Bergman R. Assessment of the prevalence of mucosal involvement in bullous pemphigoid. JAMA Dermatol. 2019;155(2):166-71. https://doi.org/10.1001/jamadermat ol.2018.5049.

26. Kridin K. Dipeptidyl-peptidase IV inhibitors (DPP4i)-associated bullous pemphigoid: Estimating the clinical profile and exploring intraclass differences. Dermatol Ther. 2020. https://doi. org/10.1111/dth.13790.

27. Izumi K, Nishie W, Beniko M, Shimizu H. A cross-sectional study comparing the prevalence of bullous pemphigoid autoantibodies in 275 cases of type II diabetes mellitus treated with or without dipeptidyl peptidase-IV inhibitors. Front Immunol. 2019;10:1439. https://doi.org/10.3389/fimmu.2019.01439.

28. Mai Y, Nishie W, Izumi K, Shimizu H. Preferential reactivity of dipeptidyl peptidase-IV inhibitor-associated bullous pemphigoid autoantibodies to the processed extracellular domains of BP180. Front Immunol. 2019;10:1224. https://doi.org/10.3389/fimmu .2019 .01224 .

29. Collin M, Ehlers M. The carbohydrate switch between pathogenic and immunosuppressive antigen-specific antibodies. Exp Dermatol. 2013;22(8):511-4. https://doi.org/10.1111/exd.12171. 